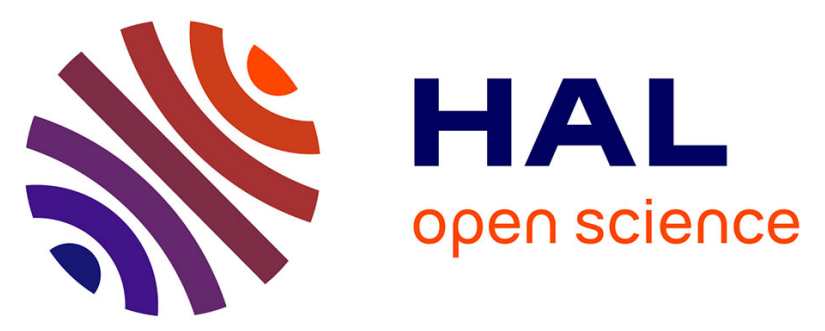

\title{
Kinematics and sensor and control systems of the fully automated facade cleaning robot siriusc for Fraunhofer headquaters in Munich
}

Norbert Elkmann, M. Lücke, Tino Kruger, Dietmar Kunst, Thomas Sturze

\section{- To cite this version:}

Norbert Elkmann, M. Lücke, Tino Kruger, Dietmar Kunst, Thomas Sturze. Kinematics and sensor and control systems of the fully automated facade cleaning robot siriusc for Fraunhofer headquaters in Munich. 6th International Conference on Field and Service Robotics - FSR 2007, Jul 2007, Chamonix, France. inria-00273008

\section{HAL Id: inria-00273008 https://hal.inria.fr/inria-00273008}

Submitted on 14 Apr 2008

HAL is a multi-disciplinary open access archive for the deposit and dissemination of scientific research documents, whether they are published or not. The documents may come from teaching and research institutions in France or abroad, or from public or private research centers.
L'archive ouverte pluridisciplinaire HAL, est destinée au dépôt et à la diffusion de documents scientifiques de niveau recherche, publiés ou non, émanant des établissements d'enseignement et de recherche français ou étrangers, des laboratoires publics ou privés. 


\title{
KINEMATICS AND SENSOR AND CONTROL SYSTEMS OF THE FULLY AUTOMATED FACADE CLEANING ROBOT SIRIUSC FOR FRAUNHOFER HEADQUARTERS IN MUNICH
}

\author{
NORBERT ELKMANN, MARIO LUCKE, \\ TINO KRÜGER, DIETMAR KUNST, \\ THOMAS STÜRZE
}

October 4, 2007

Robotic Systems, Fraunhofer Institute for Factory Operation and Automation, Sandtorstrasse 22, 39106

Magdeburg, Germany

The Fraunhofer Institute for Factory Operation and Automation IFF has developed the automatic facade cleaning robot SIRIUSc for use on the Fraunhofer-Gesellschaft's headquarters, a high-rise building in Munich, Germany. The building has a height of $80 \mathrm{~m}$, its facade an area of $4000 \mathrm{~m}^{2}$. Apart from the robot that moves along and cleans the facade, the complete, fully automated system consists of a fully automated gantry that secures, supplies energy to and above all positions the robot. Part of the project involved completely automating a standard gantry, which is an integral part of the complete facade cleaning robot system. This paper presents an overview of the significant basic functions of the robot and the gantry, emphasizing the kinematics and control and sensor systems for navigation and the cleaning sequence that employs the robot and gantry's extensive fully automatic functions. 


\section{Introduction}

The complete facade cleaning robot system consists of more than the robot alone (Figure. 1). The rooftop gantry positions the robot at the top of the facade on every pane path to be cleaned.

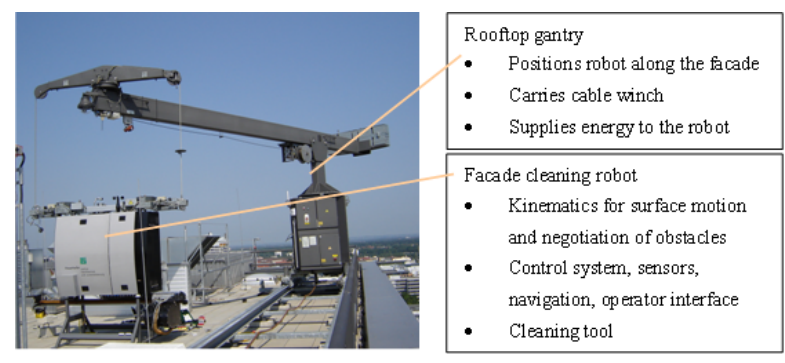

Figure 1: Rooftop gantry and facade cleaning robot atop the building

The robot then descends the facade vertically and cleans as it ascends. Four cables connected to the gantry atop the building safeguard the robot against falling. Since the cables must be taut to ensure the robot is secure, the cables are also used to position the robot as the winding and unwinding winch on the rooftop gantry moves it vertically along the building and to bear the load of the robot. Cables transmit data and supply power too. The robot is one of the first fully automatic systems of this kind used in a public setting. The robot weighs $450 \mathrm{~kg}$, the gantry $5000 \mathrm{~kg}$.

\section{Rooftop Gantry Crane}

The gantry (Figure. 2) possesses three degrees of freedom relevant for positioning the robot on the facade: Movement along the rails and two rotary cantilever arms.

Measuring systems help position the gantry on the roof to start on the pane path to be cleaned. The gantry has two asynchronous drives to move along the rails. Two encoders determine the gantry's position. The gantry is positioned by a continuous controller that reads the pane path positions taught out of a data module of the control system. Another redundant measuring system has been installed to counteract the slip.

When the encoder target position is reached, the transponders mounted on each of the pane path target positions must be matched with the transponder system. If the transponder assigned to each pane path can 


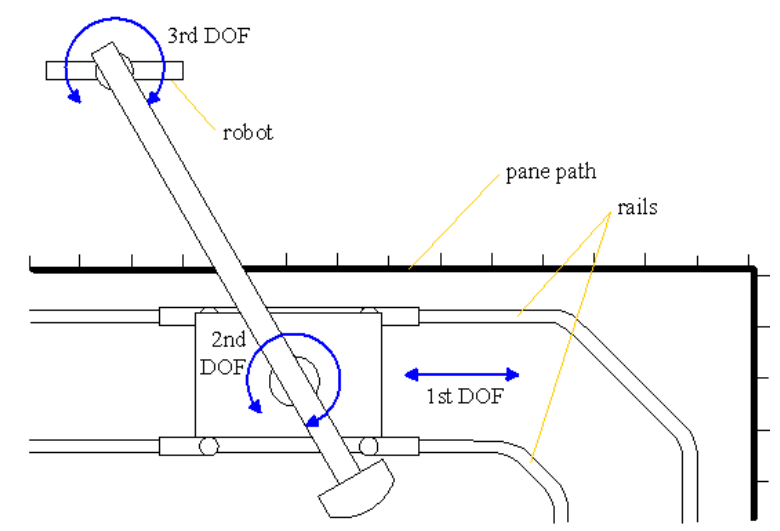

Figure 2: Gantry's degrees of freedom to position and deposit the robot on the facade

be read out successfully, the target position has been reached and the positioning of the cantilever arm can begin. Otherwise, an error code is generated and the start on the pane path must be repeated.

A synchronous movement of the two rotary cantilever arm drives sets the robot on the facade. To achieve a high level of security when positioning the robot, its orientation parallel to the facade during motion must be guaranteed. A cascaded controller in the gantry control system ensures this.

Since the rails execute a curve and do not run parallel to the facade, a particular challenge is positioning the robot and thus the gantry on the corners of the facade. The gantry's traveling mechanism (translation) and the cantilever arms (rotation) must run sequentially synchronized to prevent any collision of the robot with the facade. The gantry must proceed along the rails to reduce the robot's distance to the facade and to deposit the robot on it. Simultaneously, this negatively influences the robot's orientation plane-parallel to the facade. To simplify the positioning process, the traveling mechanism is guided in a second step to the window pane target position and then the cantilever arms are moved again so that the robot reaches its ideal position (a distance of approximately $100 \mathrm{~mm}$ and parallel to the facade). All operations are fully automated. The gantry is a standard piece of equipment that was completely automated in the project. 


\section{SIRIUSc Kinematics}

Above all, the modular kinematics (Figure. 3) ensures the robot remains in constant contact with the facade and can navigate a multitude of typical obstacles and move quickly along a facade. The kinematics is based on a structure of two pairs of linear modules, the so-called "'advanced sliding module mechanism"' $[8,9]$. Two linear modules constitute one pair that performs the same linear movement, thus ensuring secure and stable contact with the facade.

Servo drives move each of the outer or inner sucker units to the next position on a pane within a frame. The linear modules with their suckers are positioned in such a way that the suckers are located above or below the horizontal pane frames when docking onto the facade. Once a sucker unit has suctioned on, the drive's brake is deactivated and the other unit's suckers are released and retracted. The winding and unwinding of the securing cables on the gantry lift winch produces the robot's upward and downward motion on the facade. The alternation of the outer and inner sucker units with $2 \times 3$ suckers apiece produces the robot's walking motion. As the robot is lifted or lowered to the desired position, the other activated linear module pair is moved to the next free position.

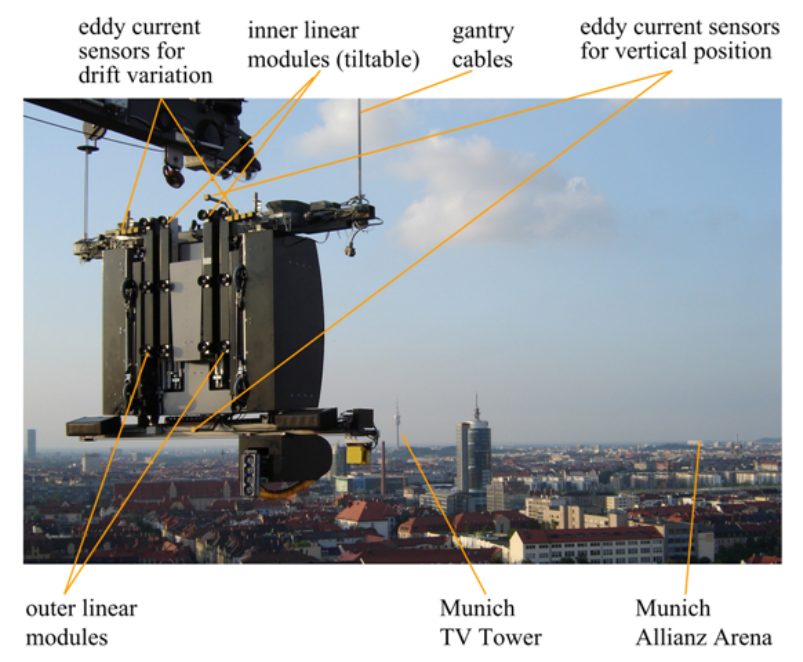

Figure 3: SIRIUSc kinematics and navigation sensors

Eddy current sensors mounted on the top and bottom of the robot's body detect and store the positions of horizontal pane frames. 
One of the requirements for outdoor operation in high winds is that the robot be able to correct its direction of motion, should it drift a bit off course. To this end, the inner pair of linear modules is tilt adjustable to enable small steering movements to keep the robot on a straight path. Two drift scanners have been mounted on the robot to control drift compensation. The scanners' job is to detect the robot's position in relation to pane frames (vertical pane jambs) in order to systematically control the inner linear modules. A drift scanner consist of $2 \times 4$ eddy current sensors (spaced $50 \mathrm{~mm}$ ), which a controller assembly switches off and on in succession. This is necessary since the eddy current sensors would otherwise interfere with one another. Depending on the robot's direction of movement and which eddy current sensors detect the metallic pane frames, the pneumatic drift compensation drive turns the inner linear guide right, to the middle or left.

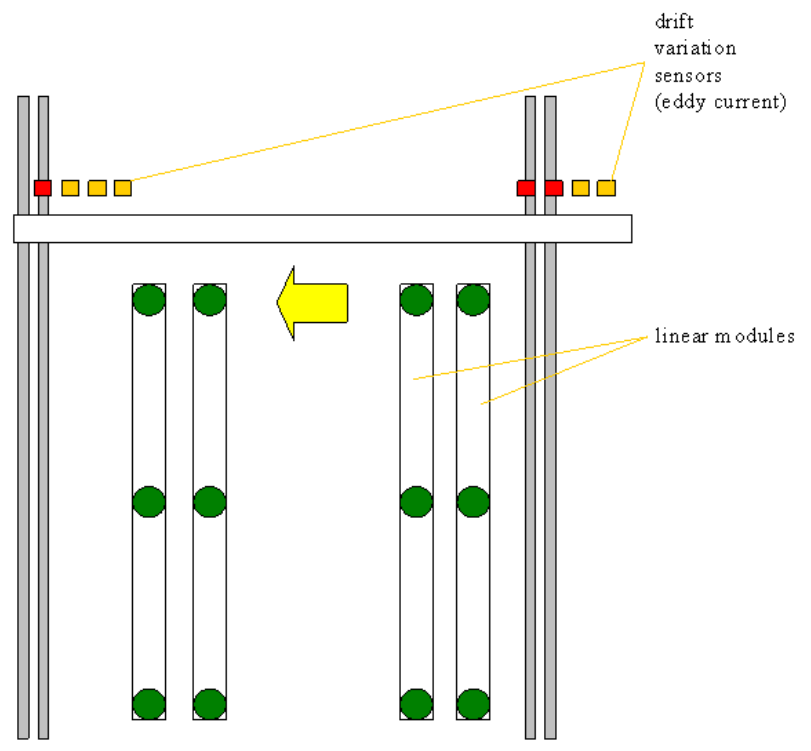

Figure 4: Schematic diagram of the detection of drift variation

The pane frames are made of various materials and profiles. This represents a special challenge. In extensive testing with various types of sensors, only novel eddy current sensors with a detection distance of 0 $50 \mathrm{~mm}$ were sufficiently reliable. None of the optical or other sensors were sufficiently reliable under the given conditions (rain, reflected sunlight on the panes). 


\section{Cleaning Sequence}

First, the gantry automatically positions the robot before the facade. Since the rails on the roof and the panes on the facade are not exactly parallel to one another, the robot must be set down supported by two tactile distance sensors mounted on it. In the process, all three of the gantry's degrees of freedom are controlled. Once the robot is located only $100 \mathrm{~mm}$ in front of the uppermost pane of the facade, four vacuum suckers with a stroke of $250 \mathrm{~mm}$ located on the robot are retracted. After the four suckers have developed a vacuum, the robot is pulled onto the pane and the robot's vacuum suckers used for moving along the facade establish the robot's contact to the facade.

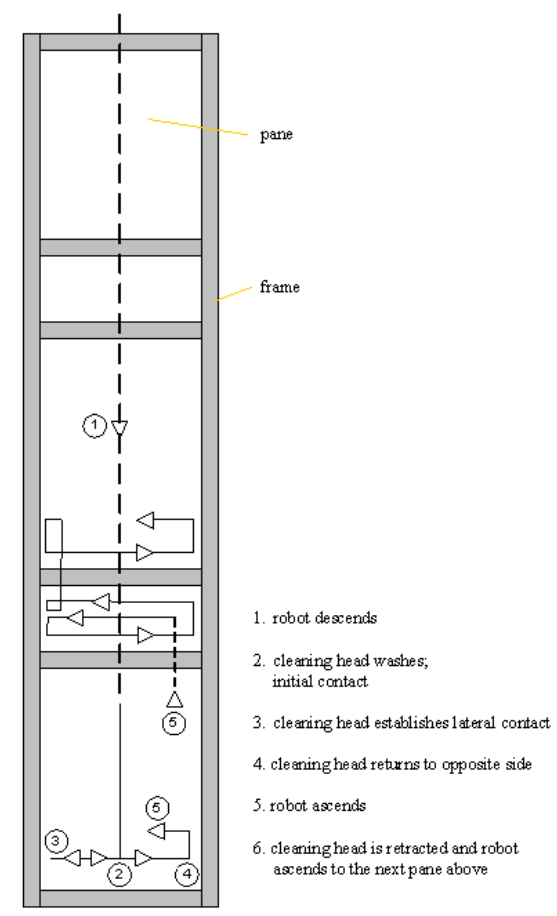

Figure 5: Robot and cleaning head motion (operation/sequence) on a pane path

The robot descends to the bottommost pane (see Section 3 on page 4) and is moved vertically by the cable winch on the gantry, the robot's suckers always being in contact with the facade. Sensors continuously register the pane frames (horizontal and vertical). Consequently, the 
direction of the robot's vertical movement is corrected and the walking algorithm is controlled. The walking algorithm positions the robot's linear modules in such a way that the maximum number of suckers is always on the facade and the suckers are always positioned on the panes and not on the frames. Once the bottom pane has been reached, the cleaning head is positioned on the pane to be cleaned (Figure. 5). The water cycle and the rotating cleaning brushes are activated. The cleaning head runs sideways until it meets the frame. Tactile sensors on all four sides of the cleaning head detect the pane frames. If the cleaning head has contact with the frame, the robot continues moving downward until the lower pane jamb has also been detected. On the lower pane jamb, the head is moved to the opposite side of the pane, cleaning the pane. Once the head has traveled back and forth one time, the robot moves upward the height of the cleaning head. When the upper pane frame is detected, the cleaning head is run once more laterally, the cleaning water suctioned up and the cleaning head retracted from the pane.

When the uppermost pane has been reached and the complete pane path cleaned, the robot disengages from the facade. Once the cleaning of a vertical pane path has concluded, the gantry moves the cleaning robot one pane width laterally.

\section{Control System and Navigation}

The heart of the system is its control system, which receives and combines sensor data and operator instructions to generate robot actions. Selected for it is stability and modularity, a programmable logic controller (PLC) is used for the robot and the gantry control system. The PLC is on board and controls the entire system. It synchronizes the walking mechanism with the trolley and cleaning head. All robot motions and actions are fully automated. The robot was programmed modularly so that it can be transferred to a large number of different facades with minimal reprogramming work. The robot does not start out with information on all the obstacles it will face in its path. Rather, it keeps track of the surface and obstacles currently under it. Sensors identify and measure obstacles and window frames. The PLC then generates the appropriate step lengths for the robot to successfully walk over obstacles and frames. The PLC ensures that vacuum suckers directly over an obstacle are not engaged while simultaneously maximizing the number of vacuum suckers in contact with the facade at any given moment. Like SIRIUS, the 
cleaning head does not require detailed information on the surface of the facade. Rather, the cleaning system has its own sensors that detect obstacles and end positions. The sensor signals are also incorporated in the onboard PLC program, which in turn uses the information to generate the necessary cleaning head movements.

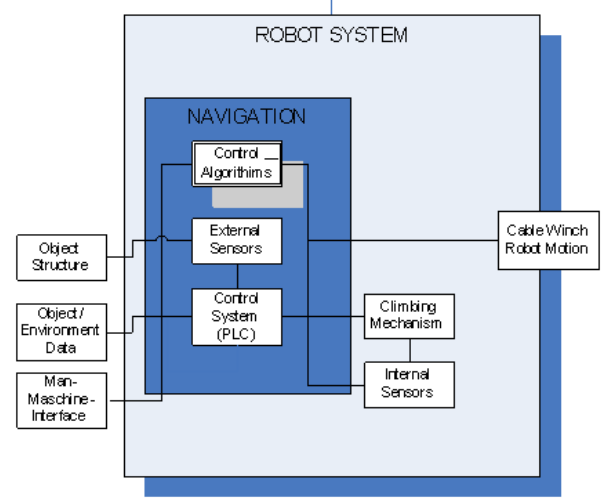

Figure 6: Control system concept

The only manual input information the robot requires before starting off on a given surface is end point data such as the height of the structure or building, off limit zones and the maximum length of obstacles. The robot automatically detects any other necessary surface information during operation. An operator master display is also located in the building so that an operator can monitor the robot's progress. A remote maintenance module allows downloading the robot's status and sensor data, uploading new program modules and executing simple operator commands such as the motion commands for a pair of linear modules. All this can be done over the Internet.

Little knowledge about the general structure of a building's surface is needed before robot movement can be generated. The input data includes end positions, moving distances and path characteristics. This a priori data is supplemented by online sensors that detect the facade surface and search for possible obstacles. In addition to identifying obstacles, the external sensor technology also corrects the direction of motion. Sensors detect where the robot must deviate from a path, e.g. girders or window and panel seals. The robot control system communicates with the building control system, making sure all windows are closed in areas being cleaned. 

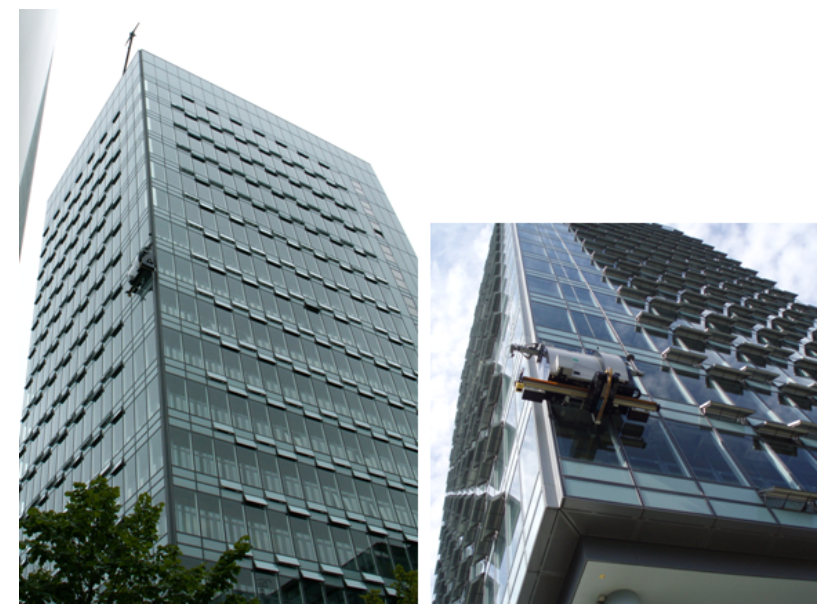

Figure 7: SIRIUSc on the high-rise building of the FraunhoferGesellschaft's headquarters in Munich

\section{Conclusion}

The Fraunhofer IFF has developed a fully automated facade cleaning robot for the Fraunhofer-Gesellschaft's headquarters in Munich. Developed for vertical facades, SIRIUSc consists of the main components of robots mechanics and kinematics, rooftop gantry, sensor systems to detect facade shape, frames and obstacles, control technology and navigation system, power supply system and integrated cleaning unit. SIRIUSc was delivered to the facility management at the Fraunhofer-Gesellschaft's headquarters in 2006. The facility management staff is now able to clean the facade without any technical support from the researchers at the Fraunhofer IFF.

\section{References}

1. S. Hirose, K. Kawabe: Ceiling Walk Climbing Robot Ninja-II. Proceedings of CLAWAR 1998, First International Symposium on Mobile, Climbing and Walking Robots, Brussels 26.-28. October, 1998, pp. 143-147

2. M. M. Cusack, J. G. Thomas: Robotics for the Inspection of Vertical Surfaces of Buildings and Structures. In 25th ISIR, pp. 287-295 
3. N. Elkmann, T. Felsch, M. Sack, T. Boehme: Modular Climbing Robot for Outdoor Operations. Proceedings of CLAWAR 1999, Second International Conference on Climbing and Walking Robots, Portsmouth 13.-15. September, 1999, pp. 413-419

4. N. Elkmann, T. Felsch, M. Sack, T. Boehme, J. Hortig, J. Saenz: Modular Climbing Robot for Service Sector Applications. In: Industrial Robot, 1999, vol. 26, number 6

5. N. Elkmann, T. Felsch, M. Sack, T. Boehme, J. Saenz: SIRIUS: Modular Climbing Robot for Facade Cleaning and Other Service Jobs. International Conference on Field and Service Robotics FSR 2001, Helsinki

6. T. Felsch, N. Elkmann, M. Sack, J. Saenz: Concepts of Service Robots for Facade Cleaning. International Symposium on Robotics ISR 2001, Seoul

7. M. Sack, N. Elkmann, T. Felsch, T. Boehme: Intelligent Control of Modular Kinematics: The Robot Platform SIRIUS. International Symposium on Intelligent Control ISIC 2002, Vancouver, Canada

8. N. Elkmann, T. Felsch, M. Sack, J.Saenz, J. Hortig: Innovative Service Robot Systems for Facade Cleaning of Difficult-to-Access Areas. International Conference on Intelligent Robots and Systems IROS 2002, Zurich

9. N. Elkmann, Lucke M., Krüger T., Kunst D, Stürze T.: SIRIUSc: Fully Automatic Facade Cleaning Robot for a High-rise Building in Munich, Germany. ISR/ROBOTIK 2006, Munich 